\title{
Intercalibración de la metodología GUADALMED. Selección de un protocolo de muestreo para la determinación del estado ecológico de los ríos mediterráneos.
}

\author{
Núria Bonada ${ }^{1}$, Narcís Prat ${ }^{1}$, Antoni Munné ${ }^{1}$, Marc Plans ${ }^{1}$, Carolina Solà 1 , \\ Maruxa Álvarez 2 , Isabel Pardo², Gabriel Moyà ${ }^{3}$, Guillem Ramon ${ }^{3}$, Manuel Toro ${ }^{4}$, \\ Santiago Robles ${ }^{4}$, Juan Avilés ${ }^{4}, \mathrm{M}^{\mathrm{a}}$ Luisa Suárez ${ }^{5}, \mathrm{M}^{\mathrm{a}}$ Rosario Vidal-Abarca ${ }^{5}$, \\ Andrés Mellado ${ }^{5}$, José Luis Moreno ${ }^{5}$, Cristina Guerrero ${ }^{5}$, Soledad Vivas ${ }^{6}$, Manuel Ortega ${ }^{6}$, \\ Jesús Casas ${ }^{6}$, Antonino Sánchez-Ortega ${ }^{7}$, Pablo Jáimez-Cuéllar ${ }^{7}$ y Javier Alba-Tercedor ${ }^{7}$
}

${ }^{1}$ Departament d'Ecologia. Universitat de Barcelona. Diagonal, 645. 08028 Barcelona.

${ }^{2}$ Área de Ecología. Universidad de Vigo. Campus Lagoas-Marcosende. 36200 Vigo.

${ }^{3}$ Departament de Biologia. Universitat de les Illes Balears. Crta. Valldemosa, km. 7.5. 07071 Palma de Mallorca.

${ }^{4}$ CEDEX. División de Ecología de los Sistemas Acuáticos Continentales. Paseo Bajo Virgen del Puerto, 3. 28005 Madrid.

${ }^{5}$ Departamento de Ecología e Hidrología. Universidad de Murcia. Campus de Espinardo. 30100 Murcia.

${ }^{6}$ Departamento de Biología Vegetal y Ecología. Universidad de Almería. Cañada de San Urbano, s/n. 04120

Almería.

${ }^{7}$ Departamento de Biología Animal y Ecología. Universidad de Granada. Campus Universitario de Fuentenueva. 18071 Granada.

\section{RESUMEN}

La aplicación de la Directiva Marco del Agua necesita que los países dispongan de una metodología de muestreo estandarizada que permita la valoración del estado ecológico de las aguas continentales. Esta metodología deberá usarse en estudios extensivos y, en general, poder ser utilizada por técnicos de formación media con un cierto grado de entrenamiento especializado. Por ello, la estandarización de protocolos de muestreo es una herramienta imprescindible para asegurar que los datos sean comparables. Asimismo en los proyectos de investigación de tipo coordinado la homogeneización de protocolos de muestreo entre equipos de investigación es básico para conseguir unas bases de datos consistentes. En el presente trabajo se presentan los resultados de un ejercicio de intercalibración de protocolos de trabajo de campo, laboratorio y uso de varios índices que pueden ser útiles para la determinación del estado ecológico de los ríos de la vertiente mediterránea peninsular y Baleares. En el ejercicio participaron 23 miembros de los seis equipos de investigación que integran los proyectos GUADALMED (HID980323-C05 y REN2001-3438-C07) y PLP/10/FS/97 de la Fundación Séneca de la Comunidad Autónoma de la Región de Murcia, y se realizó en una localidad del río Argos (Murcia).

Para la realización del estudio el grupo se dividió en cuatro subgrupos, cada uno de ellos con investigadores de los seis equipos participantes. Cada subgrupo muestreó el río en un tramo de 100 metros y cada tramo de muestreo estaba separado del anterior por 200 metros por lo menos. Se tomaron datos de características fisicoquímicas, macroinvertebrados bentónicos y bosque de ribera de acuerdo con dos protocolos de muestreo previamente acordados entre los equipos participantes. También se estandarizó la separación y contaje de los individuos en el laboratorio.

Los resultados mostraron que no existieron diferencias significativas entre subgrupos o entre los dos protocolos de muestreo y contaje testados cuando se evalúa la calidad biológica con los índices de macroinvertebrados IBMWP y IASPT, ni tampoco en el número de familias halladas entre protocolos. Si se consideraban los hábitats leníticos y lóticos por separado tampoco existían diferencias significativas entre los protocolos o los subgrupos en el valor de los índices, aunque el hábitat lenítico era mucho más diverso que el lótico. Todo ello a pesar de que existían diferencias en las comunidades encontradas en los diferentes subgrupos.

En los contajes de laboratorio se observó que a partir de 200 individuos el valor del índice IBMWP ya no varía significativamente, mientras que el valor del IASPT no varía después de contar 100 individuos.

Existieron diferencias significativas entre subgrupos a la hora de evaluar el bosque de ribera con el índice QBR, valores que no eran debidos a la subjetividad individual ya que dentro del subgrupo la variación era pequeña, sino a las diferencias en el 
hábitat en cada uno de los cuatro puntos muestreados (con cobertura arbórea muy diferente), lo que demuestra el carácter indicador local de este índice.

Con estos resultados se propone el protocolo de muestreo GUADALMED, que es el que se usó durante todo el proyecto.

Palabras clave: Protocolo, intercalibración, macroinvertebrados, estado ecológico, Directiva Marco del Agua, QBR, IBMWP, ASPT

\section{ABSTRACT}

The development of the Water Framework Directive in European countries needs standard sampling methods to evaluate the ecological status of freshwaters. Methods for use in monitoring surveys, should be suitable for non specialists. Therefore, standard protocols are necessary for the intercomparison of results from different countries. Similarly, the sampling protocols in cooperative research projects should be homogeneous in order to ensure consistent data bases. In the present work, the results of an intercalibration exercise of sampling and sorting protocols between six teams are given. Several indices potentially useful for the evaluation of ecological status of streams in the Mediterranean coast of Spain and the Balearic Islands are compared. The exercise was conducted in a sampling site in the river Argos (Murcia, Spain) and 23 researchers from six research teams from across Spain were involved. The teams formed part of the projects GUADALMED (HID98-0323-C05 and REN2001-3438-C07) and PKP/10/FS/97 funded by the "Seneca Foundation" (Murcia Regional Authority).

To perform the intercalibration exercise, the group of researchers was divided into four subgroups, each of them including researchers from the 6 teams participating in the exercise. Each subgroup took samples from a stream $100 \mathrm{~m}$ reach. Each sampling reach was separated from the previous by, at least, $200 \mathrm{~m}$. Water physico-chemistry, and macroinvertebrate and riparian habitat information were collected, following two sampling protocols previously agreed upon by all teams. Procedures and other details for sorting and counting of macroinvertebrates in the laboratory were previously fixed, too.

The results show that no significant differences exist, between the two sampling, sorting and counting protocols, nor between subgroups in the evaluation of biological quality of the stream using the indices IBMWP and IASPT (former BMWP' and ASPT'). No significant differences existed in number of macroinvertebrate families found using both protocols. When lenitic and lotic habitats were considered separately, no significant differences in the index values were found between protocols or subgroups either, although the lenitic habitat was more diverse than the lotic one.

In the laboratory, the value of IBMWP did not change significantly when estimated from 200 or from more than 200 individuals, while 100 individuals were enough to stabilize the value of IASPT.

Significant differences were found between subgroups when the riparian habitat quality was evaluated using the index QBR ("Qualitat del Bosc de Ribera" - Quality of Riparian Forest index). However, differences between subgroups were small, suggesting that differences between the four sampling reaches were indeed due to differences in \% vegetation cover of the riparian area. This demonstrates that this index is valuable as an indicator of local changes in riparian habitat condition.

Using the results of the intercalibration exercise, a sampling protocol for project GUADALMED was established.

Keywords: Protocol, intercalibration, macroinvertebrates, ecological status, Water Frame Directive, QBR, IBMWP, IASPT.

\section{INTRODUCCIÓN}

La Directiva Marco del Agua (DMA) (D.O.C.E., 2000) prevé que los países miembros determinen el estado ecológico de las aguas continentales basándose en criterios ecosistémicos (macroinvertebrados, peces, macrófitos, bosque de ribera,...). Antes del 2016 los países de la UE deberán demostrar ante la Comisión que sus ríos y lagos están en muy buen estado ecológico. En Estados Unidos el concepto de estado ecológico (formulado de diferentes maneras: salud ecológi- ca, integridad biológica, etc.) ha representado un elemento clave en los objetivos de gestión de la calidad del agua de la administración y es recogido de forma abundante en su legislación (Karr \& $\mathrm{Chu}, 2000)$. Como consecuencia de todo ello, existen varias metodologías estandarizadas para su determinación que varían según los estados (Plafkin et al., 1989; Barbour et al. 1999; Carter \& Resh, 2001). También, en algunos países europeos se han desarrollado, en los últimos años, metodologías para evaluar el estado ecológico (Bloch, 1999; Chovanec et al., 2000; Harper et 
al., 2000). En el caso de España, a pesar de los muchos estudios existentes sobre indicadores biológicos, no hay todavía metodologías estandarizadas para su aplicación a la gestión.

Para la evaluación del estado de salud de los ríos, se utilizan en diversos países los protocolos de evaluación rápida de la calidad (Rapid Bioassessment Protocols - RBP) (Wrigth et al., 1984; Plafkin et al., 1989; Davies, 1994; Tiller \& Metzeling, 1998; Chutter, 1998; Barbour et al., 1999). Los RBP, a los que llamaremos PRECE (Protocolos Rápidos de Evaluación de la Calidad Ecológica), se fundamentan en la determinación de la integridad ecológica (Karr, 1981; 1996) mediante una caracterización del hábitat, una evaluación de calidad biológica del agua y una posterior comparación con las condiciones de referencia (Barbour et al., 1999; Resh et al., 1995; Reynoldson et al., 1997). Son, por tanto, un referente a tener en cuenta al implementar la DMA para la medida del estado ecológico.

Estos protocolos han sido diseñados para ser eficientes, efectivos, fáciles de utilizar, de costos reducidos y poder ser aplicados en regiones extensas (Resh \& Jackson, 1993; Resh et al., 1995). Ello se consigue mediante un muestreo y procesado de las muestras simplificado, evitando mínimamente la pérdida de información (Resh et al., 1995; Barbour \& Gerritsen, 1996).

Una manera de simplificar y optimizar el muestreo es disminuyendo el número de muestras por punto de muestro (Resh et al., 1995; Hewlett, 2000), ya sea integrando las comunidades de los distintos hábitats presentes (Resh et al., 1995), o muestreando "el hábitat más productivo" (Plafkin et al., 1989). Ello, a pesar de que reduce el esfuerzo de muestreo, tiene implicaciones estadísticas a tener en cuenta, pues al reducir el número de réplicas por estación, no pueden ser aplicados determinados métodos paramétricos (Hulbert, 1984; Norris, 1995). A pesar de ello, el uso de estaciones de referencia como réplicas podría evitar este problema (Norris, 1995; Resh et al., 1995).

El procesado de las muestras es un factor importarte a tener en cuenta en estos PRECE.
Así, el PRECE propuesto por Tiller \& Metzeling (1998) y Metzeling \& Miller (2001) está basado en un muestro y procesado de la muestra en el campo durante 30 minutos hasta obtener unos 200 individuos. Otros métodos son independientes del tiempo y se realizan en el laboratorio, contando unos 200 individuos previa separación de los organismos de tamaño más grande (Plafkin et al., 1989; Barbour et al., 1999). Barbour \& Gerritsen (1996) demostraron que utilizando un número fijo de organismos, los patrones de distribución que resultan son similares a los mostrados con todos los individuos. No obstante, este procedimiento de submuestreo basado en un contaje fijo puede tener implicaciones en la estimación del impacto, ya que afecta a la frecuencia y abundancia de los taxones raros en la muestra (Cao \& Williams, 1999; Cao et al., 2001) y además, presupone que los organismos tengan una distribución homogénea (Countermanch, 1996). Debido a ello, otros autores son partidarios de un submuestreo basado en una fracción fija (Cuffney et al., 1993; Vinson \& Hawking, 1996; Countermanch, 1996).

Los ríos mediterráneos están sometidos a una gran variabilidad de los caudales de manera natural que supone la temporalidad de muchos de sus cauces. Además, son numerosas las alteraciones que padecen: vertidos, regulación de caudales, alteración del bosque de ribera, movimiento de áridos,... (Prat, 1993). Debido a estas características, es posible que metodologías desarrolladas en otros países puedan no ser aplicadas correctamente en este área. El proyecto GUADALMED, en su primera fase, tiene como objetivo establecer, testar e intercalibrar una metodología de muestreo basada en un PRECE. La utilización de este tipo de protocolos permitirá, en fases posteriores, la determinación del estado ecológico del agua de forma sencilla, de tal manera que podrían ser utilizados por los gestores de la administración, cuando se aplique la DMA.

La selección del protocolo a utilizar, está basada en la experiencia de los investigadores del proyecto en los ríos mediterráneos peninsulares. 
Por ello para la calidad biológica se testaron los índices biológicos IBMWP y IASPT (hasta ahora denominados BMWP' y ASPT' según Alba-Tercedor \& Sánchez-Ortega, 1988) respecto al FBILL (Prat et al., 1999). Para la evaluación del estado del bosque de ribera se usó el índice QBR (Munné et al., 1998; Suárez-Alonso \& Vidal-Abarca, 2000; Munné et al., 2003), y para la caracterización del hábitat fluvial se aplicó un índice de diversidad de hábitat modificado de Barbour et al. (1999) y adaptado a nuestros ríos (Pardo et al., este volumen). El estudio de comunidades de algas y peces no se pudo realizar dentro del proyecto GUADALMED, aunque en la DMA serán grupos a tener en cuenta y habrá que diseñar un PRECE para estos elementos de calidad.

\section{ÁREA DE ESTUDIO}

Para la selección e intercalibración de la metodología a utilizar durante el proyecto, se selec- cionó un punto de muestreo de la cabecera del río Argos (Barranda, Murcia), afluente del río Segura por el margen izquierdo (Fig. 1). Se trata de un río intermitente de una longitud de $48 \mathrm{~km}$, una pendiente del $18.6 \%$ y con una cuenca de drenaje de unos $506 \mathrm{~km}^{2}$. En el punto seleccionado, el río tiene orden 4 y la altitud es de $780 \mathrm{~m}$. Se trata de un tramo poco eutrofizado de aguas hiposalinas, bastante alcalinas, bien oxigenadas, duras, neutras y ricas en nitrógeno, tal y como se muestra en la Tabla 1 (Vidal-Abarca, 1985). El substrato del lecho está constituido, mayoritariamente, por grava, aunque también se hallan arenas, cantos y lascas calizas. La vegetación acuática es abundante, y está dominada por Oscilatoriales, Nostocales, Nemaliales, Cladophorales, Charales y Zygnematales (Aboal, 1988; 1989). En estudios previos (VVAA, 1988) el valor medio del IBMWP hallado en esta localidad es de 92, y por tanto podríamos considerarla como estación de referencia. El muestreo se realizó en Febrero de 1999.

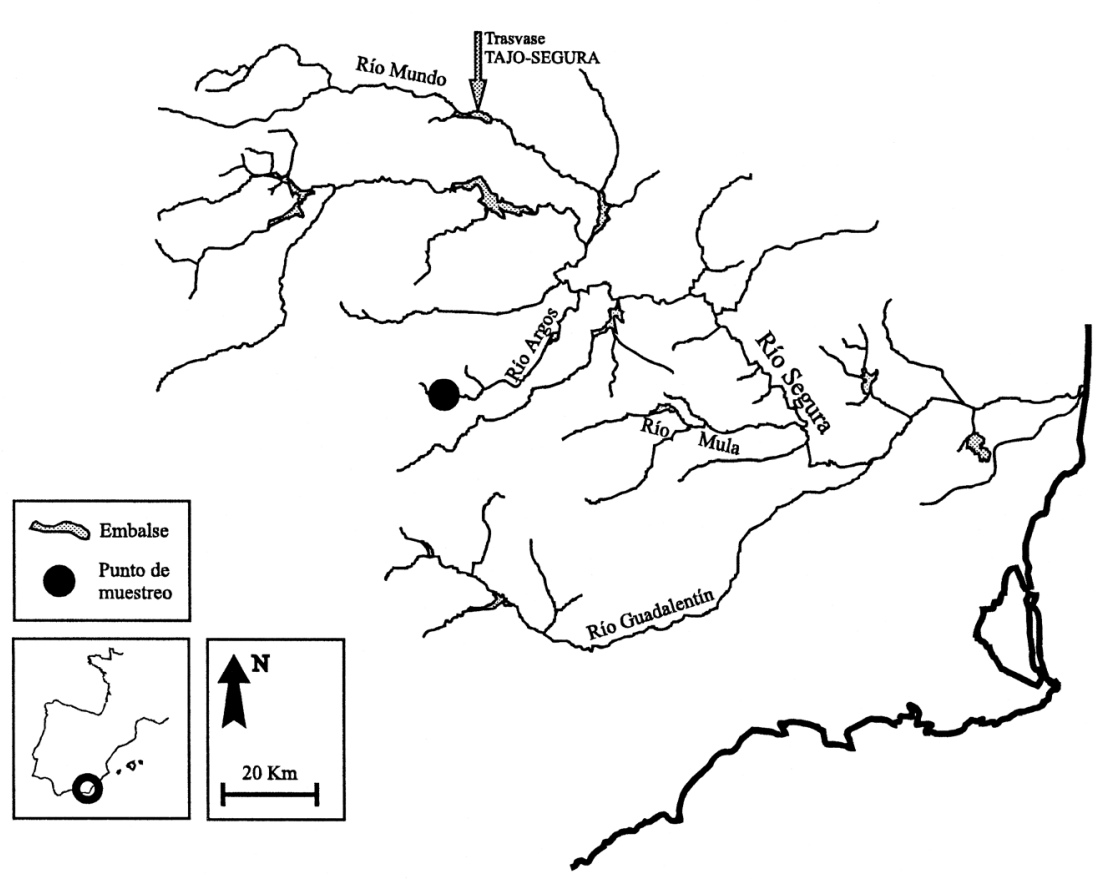

Figura 1. Cuenca del río Segura y localización del tramo de muestreo en el río Argos. Segura basin and location of the sampling reach in the Argos river. 
Tabla 1. Características físico-químicas del río Argos en la zona muestreada. Datos correspondientes a estudios anteriores (A) y a los hallados in situ con los aparatos de campo de cada grupo en el ejercicio de intercalibración (B). Physico-chemical features of Argos river in the sampling area. Data are from previous studies (A) and in situ using each laboratories resources (B).

\begin{tabular}{lcc}
\hline Variable & A & B \\
\hline $\mathrm{pH}$ & 8.6 & 8.2 \\
Salinidad $(\mathrm{g} / \mathrm{l})$ & 0.52 & \\
Conductividad $(\mu \mathrm{S} / \mathrm{cm})$ & 1203.0 & 936.0 \\
Alcalinidad $(\mathrm{meq} / \mathrm{l})$ & 6.9 & \\
Cloruros $(\mathrm{mg} / \mathrm{l})$ & 100.1 & \\
$\mathrm{O}_{2}$ disuelto $(\mathrm{mg} / \mathrm{l})$ & 11.1 & 11.5 \\
\% $\mathrm{O}_{2}$ & & 102.3 \\
Dureza $\left({ }^{\circ} \mathrm{F}\right)$ & 41.6 & \\
Calcio $(\mathrm{mg} / \mathrm{l})$ & 50.6 & \\
Magnesio $(\mathrm{mg} / \mathrm{l})$ & 70.3 & \\
Sólidos en suspensión $(\mathrm{mg} / \mathrm{l})$ & 46.5 & \\
Nitratos $(\mu \mathrm{g} / \mathrm{l})$ & 50.7 & \\
Nitritos $(\mu \mathrm{g} / \mathrm{l})$ & 1.4 & \\
Amonio $(\mu \mathrm{g} / \mathrm{l})$ & 4.7 & \\
Fosfatos $(\mu \mathrm{g} / \mathrm{l})$ & 2.2 & \\
Silicatos $(\mu \mathrm{g} / \mathrm{l})$ & 161.8 & \\
Chl-a $(\mathrm{mg} / \mathrm{l})$ & 7.5 & \\
Caudal $(\mathrm{l} / \mathrm{s})$ & & \\
\hline
\end{tabular}

\section{MATERIAL Y METODOS}

\section{Grupos de trabajo}

El estudio se planteó como una forma de homogeneizar la toma y el procesado de las muestras por diferentes equipos de trabajo, que ya tenían una experiencia previa en estudios de ríos y macroinvertebrados. Para evitar el efecto del equipo y sus costumbres en la toma y proceso de las muestras, se planteó el ejercicio de la siguiente forma:

1. Que el muestreo se realizara en el mismo río en cuatro puntos diferentes separados entre sí unos centenares de metros, con lo cual la zona era suficientemente homogénea para no presentar diferencias "a priori" de fauna o sustratos. La hipótesis fue que la fauna de macroinvertebrados no debería ser significativamente diferente en los cuatro puntos.

2. Para que cada equipo no muestreara en su forma habitual (con sus peculiaridades) y se creara un protocolo conjunto para todo el grupo GUADALMED, se dividió el conjunto de investigadores presentes en el ejercicio (todos los autores de este artículo) en cuatro grupos de trabajo, cada uno de ellos formados por miembros procedentes de diferentes equipos, de forma que no existiera en cada grupo de trabajo más de un investigador de cada equipo.

3. Se acordó el protocolo de muestreo para datos fisicoquímicos en campo, macroinvertebrados y bosque de ribera que se describe a continuación y que se aplicó a cada punto de muestreo. También se acordó el protocolo de separación y contaje de las muestras.

\section{Fisicoquímica}

Se midió la temperatura, conductividad, oxígeno y $\mathrm{pH}$, con los aparatos de campo procedentes de los diferentes equipos de trabajo (las referencias de los aparatos de campo se recogen en Toro et al. (este volumen)). Paralelamente, se tomaron mediciones de caudal usando varios modelos de velocímetro.

\section{Macroinvertebrados}

A cada grupo de trabajo se le asignó un punto de muestreo, separados por unos 100 metros. En primer lugar, cada grupo realizó un recorrido visual a lo largo de su tramo para reconocer los diferentes microhábitats a muestrear. Tras ello, se procedió al muestreo de los diferentes ambientes detectados, separando los hábitats reófilos (zonas con un mínimo de corriente) de los leníticos (zonas sin corriente). Las mangas de muestreo tenían $250 \mu \mathrm{m}$ de luz y el método utilizado fue el de barrido del sustrato (kicking).

Se establecieron dos protocolos de muestreo para ser comparados:

PROTOCOLO 1: El contenido de las mangas por cada hábitat se vertía en bateas de plástico y se iban registrando los distintos taxones hallados por observación directa, así como su rango de abundancia de acuerdo con la siguiente nomenclatura: 
$1-$ De 1 a 3 individuos.

2 - De 4 a 10 individuos.

3 - De 11 a 100 individuos.

4 - Más de 100 individuos.

Las muestras fueron observadas e identificadas en el campo, excepto los taxones más dificultosos, de los cuales se conservó uno o dos ejemplares para ser identificados en el laboratorio. Este proceso se daba por terminado cuando no se detectaban nuevos taxones en sucesivos muestreos (Alba-Tercedor, 1996; Alba-Tercedor \& Pujante, 2000).

PROTOCOLO 2: La muestra obtenida en el campo se conservaba en alcohol de $70^{\circ}$ hasta su separación e identificación en el laboratorio, con la ayuda de una lupa binocular. Se anotaban también aquellos taxones visualizados en el campo pero no capturados en la red (especialmente hemípteros y coleópteros).

Cada uno de los grupos realizó ambos protocolos en su tramo de muestreo. Para el Protocolo 2, una vez en el laboratorio, se lavaba la muestra de cada hábitat, se separaban e identificaban los individuos de mayor tamaño observables claramente sin lupa, y el resto se contabilizaba bajo la lupa, en fracciones de 50 individuos al azar, anotando el número de taxones totales para cada fracción y hábitat. Con ello, se pretende conocer el número mínimo de individuos necesarios para obtener el número óptimo de familias que indique un valor fiable de los índices biológicos.

Finalmente, los índices IBMWP, FBILL y IASPT se calcularon para cada grupo y protocolo, y los datos fueron analizados estadísticamente, mediante métodos jerárquicos de agrupación tipo Cluster y el uso de ANOVAs, previa comprobación de normalidad. Los programas estadísticos utilizados fueron Biodiversity-Pro (McAleece et al., 1997) y Statistica (StatSoft, 1999).

Los individuos recolectados fueron identificados por los diferentes especialistas presentes en el ejercicio. El listado de taxones encontrados se encuentra en el Anexo 1.

\section{Bosque de Ribera}

Todos los integrantes de cada grupo aplicaron el índice de calidad del bosque de ribera (QBR), previa explicación de cómo realizar su medida por parte de los diseñadores del índice (Munné et al., 1998). Este índice ha sido aplicado con buenos resultados en la cuenca del Segura (Suárez \& Vidal-Abarca, 2000). Los resultados del bosque de ribera y su aplicabilidad en las cuencas mediterráneas se encuentran en Suárez et al. (este volumen).

\section{Hábitat fluvial}

Se diseñó un índice para evaluar la diversidad del hábitat (Anexo 2) basado en el tipo de lecho, estructura, cobertura de la vegetación acuática, sombra en cauce, $\%$ de cada sustrato, y presencia de elementos de heterogeneidad. Este índice no tuvo un resultado satisfactorio en los muestreos posteriores, por lo cual fue substituido por otro, cuyos resultados y aplicación se presentan en Pardo et al. (este volumen).

\section{Macroalgas y macrófitos}

Las macroalgas y macrófitos más abundantes fueron identificadas por miembros de la Universidad de Murcia. Se pretendía que, durante el desarrollo del proyecto, se hiciera un inventario de los macrófitos y macroalgas de las localidades muestreadas, para con ello establecer un índice de diversidad.

\section{RESULTADOS}

\section{Fisicoquímica}

Los aparatos de campo de las diferentes instituciones obtuvieron medidas similares para cada uno de los parámetros físicos analizados (los valores medios de los parámetros medidos se muestran en la Tabla 1). Además, los dos velocímetros testados dieron valores de caudal similares $\left(10.9 \mathrm{l} / \mathrm{s}\right.$ y $\left.10.3 \mathrm{l} / \mathrm{s}^{1}\right)$. Con ello, y como era de 
esperar, cada equipo podrá medir in situ, temperatura, $\mathrm{pH}$, conductividad, oxígeno y caudal, y los resultados serán comparables.

\section{Macroinvertebrados: Selección del índice biológico}

Para cada uno de los protocolos se calculó el IBMWP para las muestras integradas (reófilo y lenítico) y el FBILL para las reófilas. Los datos, que se representan en la figura 2, muestran como para el FBILL no existen diferencias entre protocolos. Además, se observa como en cualquiera de

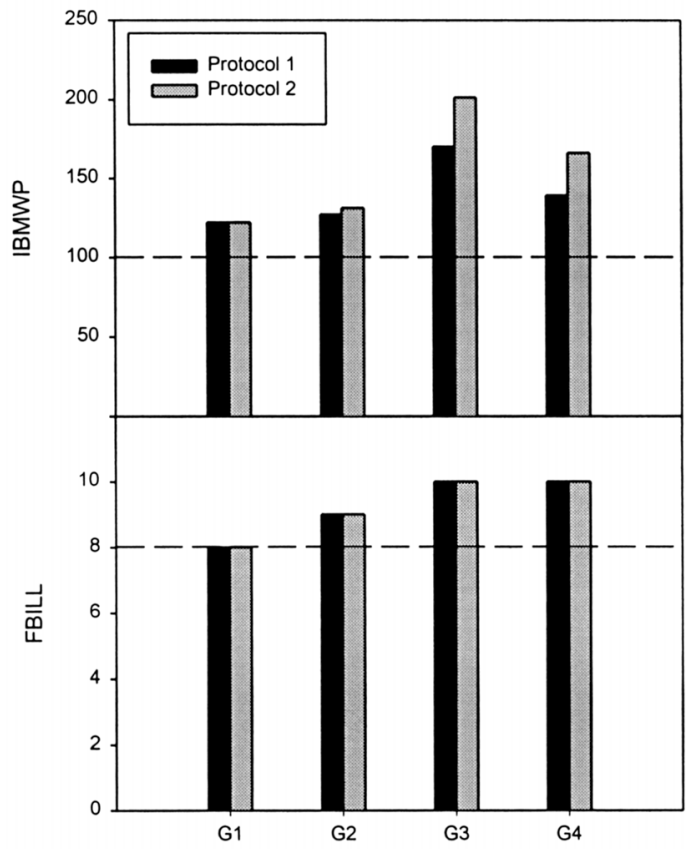

Figura 2. Valores del IBMWP y FBILL para todos los grupos de muestreo y ambos protocolos. El IBMWP se ha calculado mediante la composición de la muestra integrada $(\mathrm{R}+\mathrm{L})$ mientras que el FBILL únicamente con la del hábitat reófilo (R). Las líneas discontinuas muestran los valores de cada índice a partir del que se considera una calidad del agua "Muy Buena" (>100 para el IBMWP y entre 8-10 para el FBILL) (G1, G2, G3 y G4 = Grupos 1, 2, 3 y 4). Values of IBMWP and FBILL for all sampling groups and using both protocols. The IBMWP index has been estimated from the biological community in the integrated sample $(R+L)$, while the FBILL index used individuals collected from the lotic habitat $(R)$. The broken lines show the values taken by each biotic index. Water quality can be considered of "Very good" quality (>100 in the IBMWP scale and 8-10 in the FBILL scale) (G1, G2, G3 and G4 = Groups 1, 2, 3 and 4).

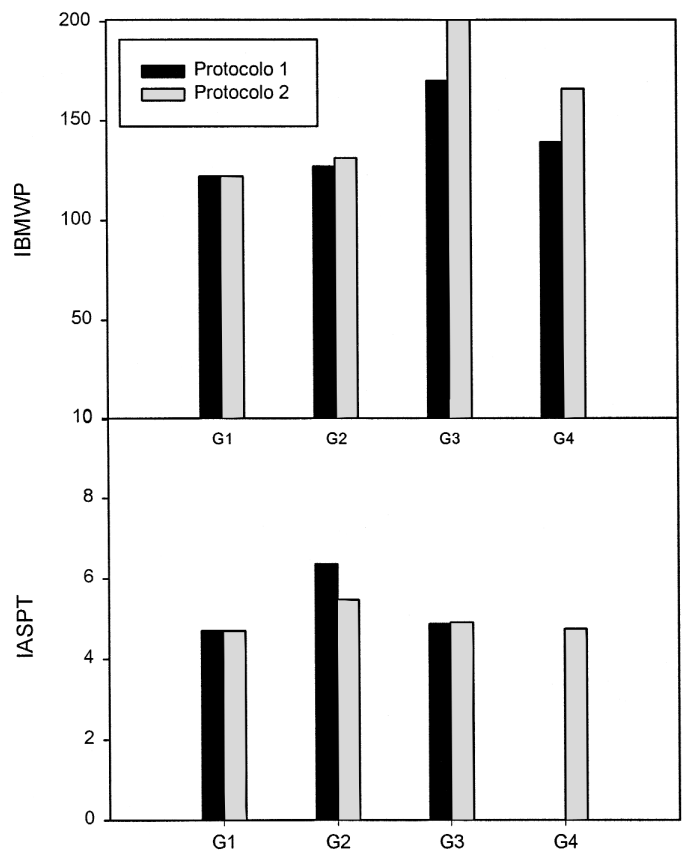

Figura 3. Valores de IBMWP y IASPT según el Protocolo 1 y 2. IBMWP and IASPT values following Protocols 1 and 2.

los grupos de muestreo se alcanzan tanto para el FBILL y el IBMWP la "Muy buena calidad", con lo que ambos índices podrían ser utilizados. No obstante, debido a que en algunos casos los ríos mediterráneos pueden tener un hábitat reófilo muy reducido, se decidió aplicar el IBMWP en lugar del FBILL. De esta manera, también se dispondría de la composición de macroinvertebrados de los hábitats leníticos, muy importante desde el punto de vista faunístico.

\section{Macroinvertebrados: Efecto del protocolo de muestreo}

Se realizó un análisis de la varianza para comparar el número de familias y los valores del IBMWP y IASPT para cada protocolo de muestreo y hábitat. Se utilizaron los datos de cada grupo de trabajo como réplicas $(n=4)$. Según los resultados, no existen diferencias significativas por lo que al IBMWP y al IASPT se refiere entre el Protocolo 1 y $2(\mathrm{p}=0.4884$ y $\mathrm{p}=0.5924)$ (ver Fig. 3). El número total de 
Tabla 2. Familias no registradas en el campo y halladas en el laboratorio utilizando el Protocolo 2, y su valor de IBMWP correspondiente. Families not registered in the field but found in the laboratory using Protocol 2, and their corresponding IBMWP values.

\begin{tabular}{lcc}
\hline Taxon & Grupo & IBMWP \\
\hline Oligochaeta & G-3 & 1 \\
Ostracoda & G-3 & 3 \\
Lymnaeidae & G-3 & 3 \\
Caenidae & G-1 y G-4 & 4 \\
Hydroptilidae & G-3 & 6 \\
Psychomyidae & G-3 & 8 \\
Helodidae & G-4 & 3 \\
Sericostomatidae & G-3 & 10 \\
Elmidae & G-3 & 5 \\
Simuliidae & G-4 & 5 \\
Ephydridae & G-4 & 2 \\
Psychodidae & G-4 & 4 \\
Stratiomyidae & G-2 & 4 \\
Limoniidae & G-3 & 4 \\
Ceratopogonidae & G-3 & 4 \\
\hline
\end{tabular}

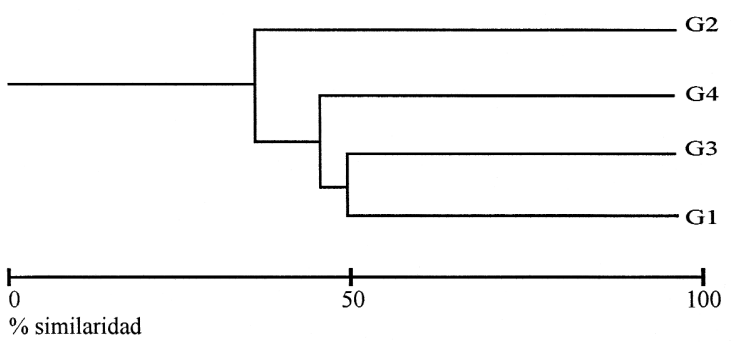

Figura 4. Dendrograma de la composición taxonómica de macroinvertebrados hallada por cada grupo, utilizando el método de Jaccard. Dendrogramme of the macroinvertebrate taxa composition found by each team, using Jaccard's method.

familias halladas tampoco difiere entre protocolos $(\mathrm{p}=0.4832)$ ni para cada uno de los hábitats $(\mathrm{p}($ lenítico $)=0.8351 \mathrm{y} \mathrm{p}($ reófilo $)=0.7608)$.

A pesar de que no se hallaron diferencias significativas para el número de familias entre el protocolo 1 y 2 , mediante este último se registraron algunos taxones adicionales en cada uno de los grupos (Tabla 2), algunos de ellos de difícil visualización e identificación en el campo (como los dípteros pequeños u ostrácodos). El grupo de trabajo 2 fue el que experimentó una mayor similitud entre los taxones obtenidos según los protocolos 1 y 2 .

\section{Macroinvertebrados: Efecto del grupo de trabajo}

Con los taxones hallados en campo y laboratorio conjuntamente, y para cada grupo, se construyó una matriz de presencia/ausencia. Dicha
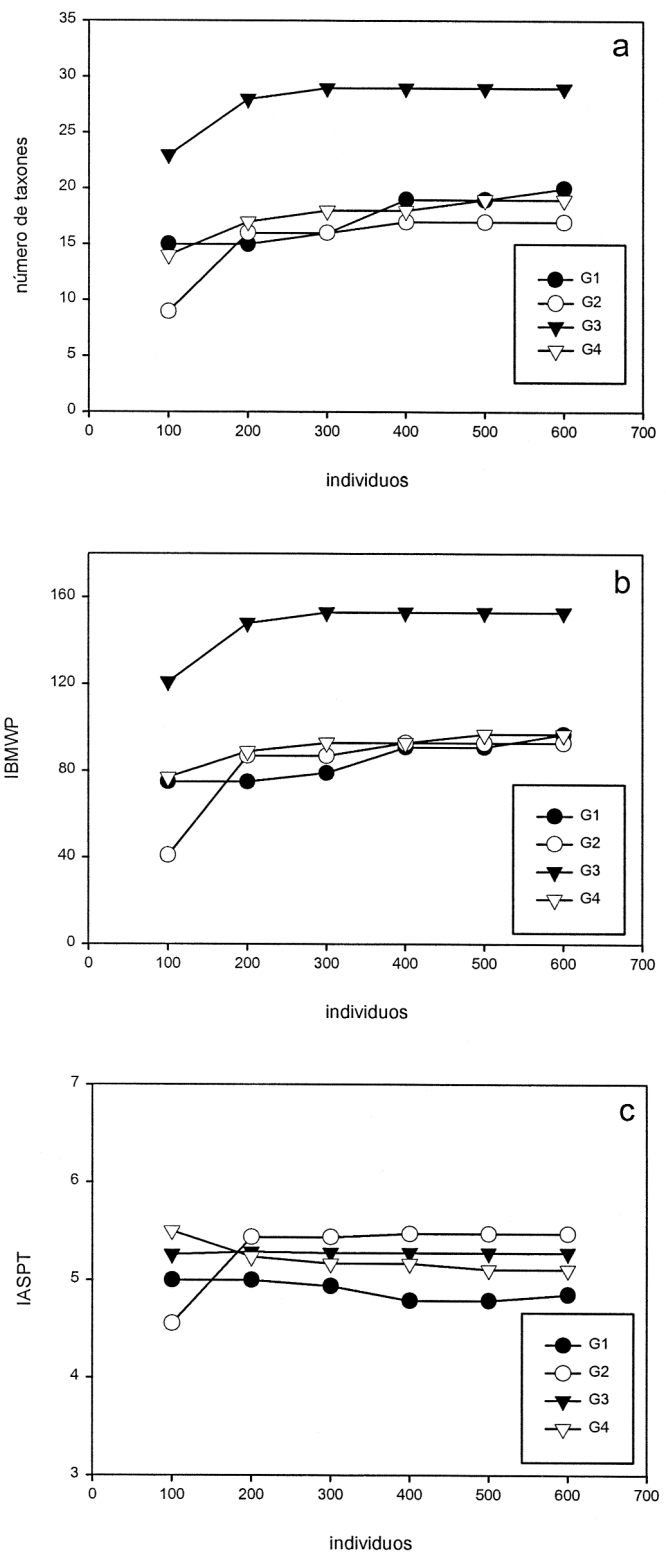

Figura 5. Número de taxones, IBMWP y IASPT acumulados para cada centenar de individuos y para cada grupo de trabajo. Number of taxa, cumulative IBMWP and IASPT for each hundred of individuals and for each working team. 
matriz, se utilizó para la obtención de un clúster con el fin de conocer las similitudes entre grupos de muestreo y testar la intercalibración. El coeficiente de similitud utilizado fue el de Jaccard que excluye las doble ausencias, y el dendrograma se representa en la figura 4 . La mayor similitud entre grupos, se encuentra entre el grupo 1 y 3 (50\%), mientras que el grupo 2, encontró una comunidad algo más diferente a los demás (39\% de similitud). No obstante, el valor de IBMWP en campo y en laboratorio de este grupo es muy parecido al hallado por los demás (Fig. 3). Ello nos indicaría que la comunidad encontrada por el grupo 2 es más pobre pero con valores ecológicos superiores, hecho que podría estar relacionado con una mayor presencia del hábitat reófilo en el tramo muestreado por este grupo.

\section{Macroinvertebrados: Efecto del contaje de individuos}

En la figura 5 se representa el número de taxones, el valor de los índices IBMWP y IASPT calculados y acumulados para cada centenar de individuos contados en ambos hábitats (50 correspondientes al reófilo y 50 al lenítico). Se observa un incremento del número de familias y del valor del IBMWP al pasar de 100 a 200 individuos contados, estabilizándose a partir de este punto. Para el IASPT, el valor obtenido para cada grupo con 100 individuos, en general no varía al incrementar el esfuerzo de contaje. Ello reforzaría el buen uso de este índice debido a su propiedad conservativa, respecto a los demás. Los análisis de la varianza realizados para testar las diferencias entre grupos para cada una de las variables muestran la presencia de diferencias significativas entre grupos $(p=0.000$ para el número de familias, $\mathrm{p}=0.000$ para el IBMWP y $\mathrm{p}=0.008$ para el IASPT). Según ello, el grupo 3 es el que posee más número de familias y por tanto un mayor IBMWP pero, en cambio, el valor del IASPT es intermedio, lo que indicaría que el incremento de familias se ha hecho mediante la adición de taxones tanto del hábitat reófilo como del lenítico, hecho relacionado con
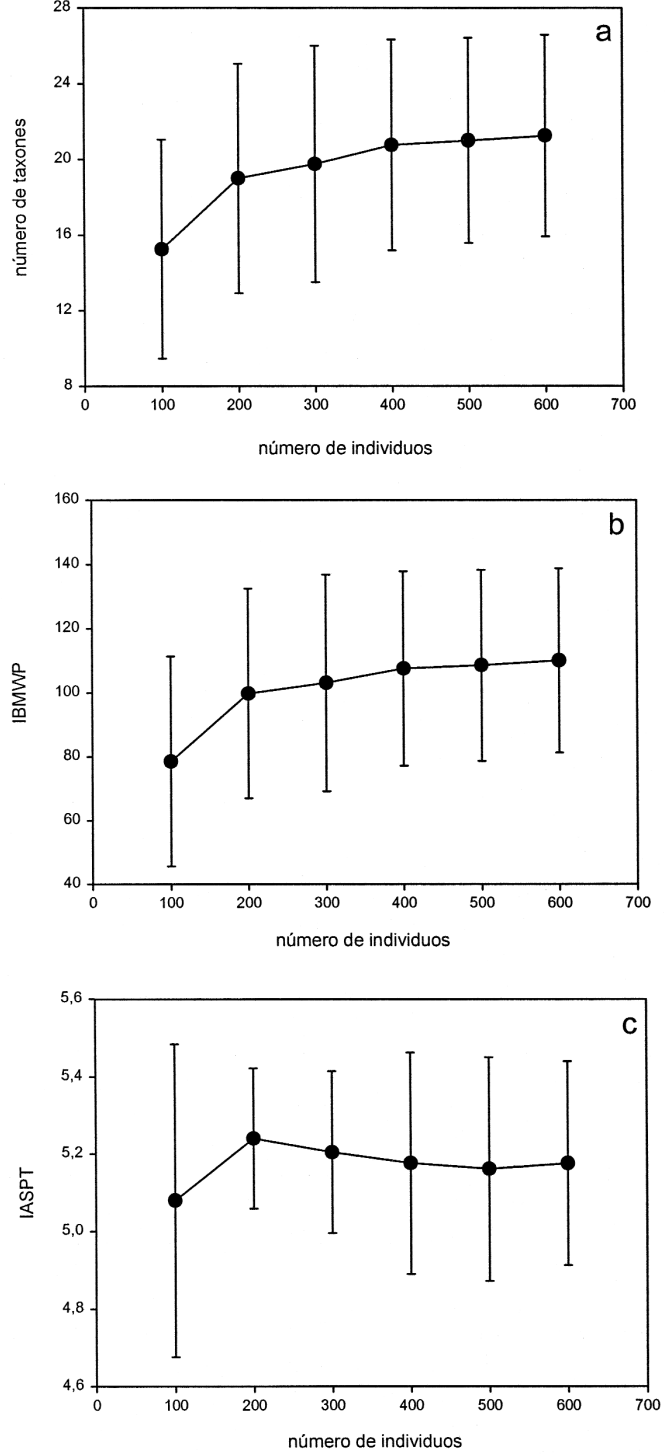

Figura 6. Valores medios y desviación estandar del número de taxones, IBMWP y IASPT hallados en función de la fracción de la muestra contabilizada para todos los grupos de trabajo. Mean values and standard deviation of the number of taxa, IBMWP and IASPT versus the fraction of the sample counted by all sampling groups.

un mayor y más intensivo muestreo del tramo respecto al realizado en los demás grupos. Para el grupo 2, que tal y como ya se ha comentado tiene una comunidad más pobre que los demás grupos, se observa como a pesar de poseer un menor número de familias y un IBMWP bajo, 


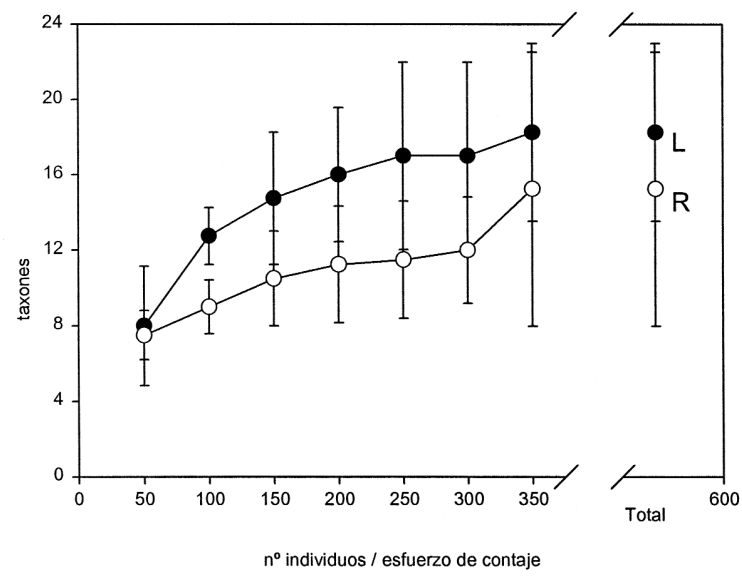

Figura 7. Número de taxones hallados en función de la fracción de la muestra contabilizada para todos los grupos, pero según el hábitat reófilo (R) y lenítico (L). Number of taxa found versus the fraction of the sample counted by all sampling groups, separately in lotic (R) and lenitic (L) habitats.

hallamos un IASPT más elevado que en los demás grupos, hecho que reforzaría la idea de que el hábitat de este grupo era mayoritariamente reófilo. Así pues, parece ser que las diferencias existentes entre grupos están relacionadas con el hábitat disponible para ser muestreado.

Al representar los valores medios y las desviaciones estándar del número de taxones, el IBMWP y el IASPT para todos los grupos y hábitats conjuntos (Fig. 6) se observa un incremento de los valores medios de cada variable entre los 100 y 200 individuos, a pesar de las elevadas desviaciones estándar, debidas a la variabilidad de los hábitats disponibles. Esto último supone que en ningún caso, se encuentren diferencias significativas entre el número de individuos medidos $(\mathrm{p}=0.6912$ para el número de familias, $\mathrm{p}=0.7293$ para el IBMWP y $\mathrm{p}=0.9788$ para el IASPT), lo que indicaría que contando 100 individuos es suficiente para obtener un número de individuos, un IBMWP y IASPT óptimos.

Al observar las diferencias entre los dos hábitats (Fig. 7) observamos un incremento asintótico del número de taxones para cada fracción de individuos contada y para ambos hábitats. El análisis de la varianza realizado para comparar los dos hábitats nos indica diferencias signi-
Tabla 3. Taxones exclusivos del hábitat reófilo y lenítico. Taxa only found in lotic and lentic habitats.

\begin{tabular}{ll}
\hline Exclusivos R & Exclusivos L \\
\hline Hydracarina & Coenagrionidae \\
Perlidae & Corduliidae \\
Aeshnidae & Cordulegasteridae \\
Helodidae & Dryopidae \\
Psychomyiidae & Haliplidae \\
Psychodidae & Hydraenidae \\
Simuliidae & Hydrophilidae \\
Stratiomyidae & Naucoridae \\
Tabanidae & Nepidae \\
& Notonectidae \\
& Polycentropodidae \\
& Ephydridae \\
& Glossiphoniidae \\
\hline
\end{tabular}

ficativas entre el hábitat lenítico y el reófilo $(\mathrm{p}<0.005)$, siendo el primero más diverso en número de taxones que el segundo. Además, parece ser que en la localidad muestreada al aumentar la fracción contada existe un mayor incremento de los taxones leníticos que los reófilos, ya que la pendiente es mayor en el primer hábitat que en 1 segundo. En la Tabla 3 se muestran por grupos los taxones hallados exclusivamente en un hábitat y en otro. En general se observa que las familias más características del hábitat reófilo son aquellas especializadas en ese hábitat como los simúlidos, helódidos, psicómidos,... Lo mismo sucede en la zona lenítica, donde los heterópteros, odonatos y coleópteros son los más abundantes.

\section{Bosque de ribera: Efectos del grupo}

Los análisis de la varianza realizados para testar diferencias entre grupos por lo que se refiere a los valores del QBR, mostraron que éstas son significativas $(\mathrm{p}<0.001)$. Ello puede indicar dos cosas. Por una parte, que el bosque de ribera no era uniforme en los cuatro puntos muestreados y por ello los valores fueron diferentes (en algunas zonas había más árboles que en otras y esto es lo que está reflejado en el valor del índice). Por otra parte podría indicar una subjetividad en la interpretación del método, pero esto no es así tal y 
Tabla 4. Valores del QBR por grupos y observadores. $Q B R$ values reaches by different teams and observers.

\begin{tabular}{rll}
\hline Grupo & Valor QBR de cada investigador & Media \\
\hline I & $40-40-20-25-30$ & 31 \\
II & $70-70-50-70-75$ & 67 \\
III & $40-30-30-35-25$ & 32 \\
IV & $40-45-55-60-55-50$ & 50 \\
\hline
\end{tabular}

como se demuestra en la Tabla 4, donde se indican los valores de QBR de cada observador. Según esta tabla, aunque existen diferencias entre los diferentes observadores, los cambios de rangos de calidad son de un solo nivel como máximo en cada grupo, lo que ratifica la validez del método y las observaciones sobre la relativa baja subjetividad del mismo, expuestas en Munné et al. (2003).

\section{Macroalgas y macrófitos}

En la Tabla 5 se muestran los macroalgas y macrófitos más abundantes en el tramo muestreado, así como su forma biológica en la que se hallan.

\section{DISCUSIÓN}

Varios autores han estudiado el efecto de la simplificación de los PRECE en la pérdida de infor- mación, ya sea por una reducción del número de muestras, del área muestreada, separación en campo frente a laboratorio, del esfuerzo de contaje o de la suficiencia taxonómica (Resh \& Unzicker, 1975; Marchant et al., 1995; Resh et al., 1995; Plafkin et al., 1989; Barbour \& Gerritsen, 1996; Barbour et al., 1999; Smith et al., 1999). El proyecto GUADALMED en su primera fase, tiene un área de aplicación relativamente grande (desde el río Besós en Cataluña hasta el Guadalfeo en Granada), y además, pretende ampliar su zona de estudio en el futuro (segunda fase proyecto GUADALMED). Por lo tanto, protocolos de tipo PRECE han de ser contemplados para, con ello, poder ser utilizados también por la administración, en la implantación de la DMA en España. En el presente estudio se han comparado dos tipos de protocolos para la evaluación de la calidad del agua mediante macroinvertebrados, para que el más óptimo, en términos de rapidez y respecto a la información proporcionada, fuera seleccionado.

Al implementar una metodología de muestreo en una zona donde no existe, lo primero que se debería hacer es testar varias, seleccionar alguna y adaptarla de manera conveniente, en caso de que fuera necesario, ya que no todas pueden ser útiles (Rosenberg \& Resh, 1993; Resh et al., 1995; Hill et al., 2000; Wrigth et al., 2000). En el

Tabla 5. Listado de los macrófitos y macroalgas hallados en el tramo de río estudiado. List of macrophytes and macroalgae found in the studied river section.

\begin{tabular}{|c|c|c|c|c|c|}
\hline División & Clase & Orden & Familia & Género/especie & Forma biológica \\
\hline \multirow[t]{4}{*}{ Cyanophyta } & Cyanophyceae & Oscillatoriales & Oscillatoriaceae & $\begin{array}{l}\text { Oscillatoria sp. } \\
\text { Lyngbya sp. }\end{array}$ & $\begin{array}{l}\text { Pecton } \\
\text { Pecton }\end{array}$ \\
\hline & & Nostocales & Rivulariaceae & Rivularia sp. & Pecton \\
\hline & & & & Homeothrix sp. & Pecton \\
\hline & & & Scytonemataceae & Tolypothrix sp. & Plocon \\
\hline Heterokontophyta & Bacilliarophyaceae & Naviculales & Naviculaceae & Cymbella sp. & Pecton \\
\hline Rhodophyta & Florideophyceae & Nemaliales & Batrachospermaceae & $\begin{array}{l}\text { Batrachospermum } \\
\text { moniliforme }\end{array}$ & Plocon \\
\hline \multirow[t]{5}{*}{ Chlorophyta } & Cladophorophyceae & Cladophorales & Cladophoraceae & $\begin{array}{l}\text { Cladophora } \\
\text { glomerata }\end{array}$ & Plocon \\
\hline & & & & $\begin{array}{l}\text { Rhizoclonium } \\
\text { hieroglyphicum }\end{array}$ & Plocon \\
\hline & Charophyceae & Charales & Characeae & Chara vulgaris & Rizófito-limnófito \\
\hline & Zygnematophyceae & Zygnematales & Zygnemataceae & Spirogyra sp. & Plocon \\
\hline & & & & Zygnema sp. & Plocon \\
\hline
\end{tabular}


presente estudio se han testado dos protocolos, uno exclusivamente basado en campo, y otro en el laboratorio. En ambos protocolos se aplica un muestreo no cuantitativo mediante la técnica de barrido. En la literatura, existen numerosos métodos para la recolección de macroinvertebrados en función del tipo de río y de los objetivos planteados (ver Rosenberg, 1978; Elliot \& Tullett, 1978; 1983). El método de barrido es un método no cuantitativo, sencillo y útil cuando el uso de muestras cualitativas no es un problema y está recomendado en los estudios de calidad biológica (Storey et al., 1991; Metzeling \& Miller, 2001). Además, los métodos no cuantitativos pueden ser utilizados en sustratos donde las técnicas cuantitativas no son aplicables pero que al contener taxones característicos es necesario muestrearlos (Chessman \& Robinson, 1987). El tipo de datos a utilizar (cualitativos o cuantitativos) es irrelevante para mostrar los patrones de las comunidades (Gauch, 1982), aunque a pequeña escala y en función de los objetivos planteados, los datos cuantitativos son necesarios (Marchant, 1990).

Varios autores sugieren que los resultados obtenidos en muestreos fragmentados por hábitat, y con una posterior integración de los resultados, aportan información redundante $\mathrm{y}$, por tanto, el muestreo de uno sólo sería suficiente para determinar si existe alguna perturbación y sus efectos (Plafkin et al., 1989). Nuestros resultados contradicen estos estudios, ya que los hábitats leníticos suponen un aporte de numerosos taxones distintos a los reófilos, principalmente coleópteros y heterópteros que, a pesar de que no tienen puntuaciones elevadas en el IBMWP, son muy abundantes en los ríos mediterráneos temporales (Rieradevall et al., 1999; Mellado et al., 2002), y por tanto reducen el valor del IASPT incluso en condiciones de referencia. Por otra parte, en ríos de montaña (Sierra Nevada, Pirineos), la relevancia del hábitat lenítico es mucho menor, y la mayor diversidad se encuentra en el reófilo (Carter \& Fend, 2001). Es por ello, que es necesario realizar un muestreo integrando los dos hábitats. Además, debido a la elevada variación de los caudales en los ríos mediterráneos, los hábitats presentes pueden variar anual e interanualmente
(Bonada, 2003). Así pues, una muestra integrada reófilo-lenítico puede dar una buena información sobre la comunidad de los ríos estudiados (Kerans et al., 1992; Cuffney et al., 1993), a pesar de requerir un mayor esfuerzo. Así pues, a pesar de que el IBMWP (Alba-Tercedor \& SánchezOrtega, 1988) y FBILL (Prat et al., 1999) podrían ser igualmente aplicables, debido a la importancia que el hábitat lenítico puede tener en los ríos mediterráneos, sobretodo en algunas épocas de muestreo, se decidió aplicar únicamente el IBMWP, sin que ello indicara un mal funcionamiento del FBILL en los ríos mediterráneos. En este sentido, algunos autores (Rico et al., 1992; Prat et al., 1997) muestran elevadas correlaciones entre los valores del BILL (índice en el que se basa el FBILL) con el IBMWP, indicando con ello la elevada aplicabilidad de el primero en los ríos peninsulares. Por su parte, el BILL (a nivel de género o familia) está altamente correlacionado con el FBILL (basado exclusivamente en familias para simplificar su aplicación), tal y como se indica en Prat et al. (1999).

Para determinar la diversidad del hábitat, Hannaford et al. (1997) compararon la capacidad de un grupo de estudiantes con experiencia y otro sin ella. Los resultados indicaron que el grupo más experimentado obtenía resultados más precisos, muy distantes a los obtenidos en el otro grupo. De la misma manera, para obtener resultados de macroinvertebrados en el campo comparables con los hallados en el laboratorio, la experiencia y aprendizaje también son importantes. Estudios similares al presente realizados por personal cualificado suministran datos para la separación en el campo del $76 \%$ de las familias presentes en un tramo fluvial, frente al $90 \%$ del total de las familias obtenido en la separación en laboratorio (Smith et al., 1999). Aún así, ello implicaría que la selección del Protocolo 1, a pesar de ser rápido $\mathrm{y}$ efectivo, requeriría un esfuerzo previo de aprendizaje, hecho importante si quiere ser utilizado por los gestores de la administración. En este trabajo, donde cada grupo estaba constituido por un miembro de cada institución, con una experiencia similar en la identificación en campo de los macroinvertebrados, las diferencias 
entre las comunidades halladas por cada uno (de hasta un $60.08 \%$ ) deberían ser debidas a cambios en el hábitat presente en cada punto y no a la falta de experiencia. Ello parece ser así en nuestros resultados ya que en algunos grupos encontramos en campo valores más elevados de IBMWP con IASPT bajos debido a la dominancia del hábitat lenítico, mientras en otros, los valores del IBMWP son bajos pero el IASPT es elevado, hecho que podría estar relacionado con una mayor presencia del hábitat reófilo. Es interesante observar como a pesar de estas diferencias, existe una gran similitud en el rango de IBMWP hallado en cada grupo.

El número de taxones encontrados en una muestra aumenta asintóticamente, a medida que lo hace el esfuerzo de contaje de la misma (Courtemanch, 1996; Vinson \& Hawkins, 1996). Ello supone que algunos autores (Cuffney et al., 1993; Countermanch, 1996; Vinson \& Hawkins, 1996) critiquen el método de contaje fijo para determinar diversidad de la muestra. No obstante, otros autores demuestran su gran utilidad (Plafkin et al., 1989; Barbour \& Gerritsen, 1996; Tiller \& Metzeling, 1998; Barbour et al., 1999; Metzeling \& Miller, 2001) a pesar de que el número de organismos utilizado es variable tal y como se muestra en Carter $\&$ Resh (2001). Según los resultados obtenidos en este estudio, fracciones de unos 100 individuos serían suficientes para obtener un número de familias, IBMWP y IASPT óptimos y no significativamente diferentes a los hallados contabilizando toda la muestra. No obstante, y según la figura 5, para las variables número de taxones y IBMWP hallamos un incremento en todos los grupos entre la fracción de 100 y 200, hecho que estadísticamente parece no ser significativo al juntar todos los grupos debido a la gran desviación estándar. Así pues, consideramos que para hallar un IBMWP y número de taxones óptimo se requerirían 200 individuos en lugar de 100. De esta manera, a pesar de que el número de taxones y el IBMWP sigan aumentando ligeramente utilizando más de 200 individuos, el rango de calidad permanece estable. El IASPT en cambio, parece mantenerse estable incluso a partir de 100 individuos (Fig. 5). Así pues, tal y como vemos en estos resultados, el IASPT es la métrica más conservativa ya que obtenemos un IASPT válido solamente contabilizando 100 individuos. Ello indicaría la gran utilidad de este índice en estudios donde el tiempo y los costos son limitados.

En Marchant et al. (1995) y Marchant (1999) se sugiere que la exclusión de los taxones raros no implican una pérdida de información ecológica. No obstante, otros autores (Cao et al., 2001) inciden en la importancia de las especies raras para obtener una buena estima de la calidad (Cao \& Williams, 1999). En nuestro caso, si bien no existen diferencias significativas entre el IBMWP y el IASPT para todos los grupos, hecho atribuible a la elevada desviación estándar, al analizar los datos para cada uno de los grupos y observar que se necesitan 200 individuos para estabilizar el IBMWP pero solamente 100 para el IASPT, estos 100 individuos de más contendrían aquellos taxones raros y poco abundantes, pero significativos para incrementar el índice biológico, aunque no para el IASPT. Ello indicaría que los taxones raros (poco abundantes y frecuentes) que puedan encontrarse entre 100 (número óptimo de IASPT) y 200 (número óptimo del IBMWP) son importantes ya que suponen un aumento significativo del IBMWP. Además, las especies raras, aunque poco abundantes y frecuentes, serían las clave para determinar los efectos de las perturbaciones a un nivel más detallado ya que normalmente están caracterizados por presentar unos rangos ecológicos muy determinados (Cao \& Williams, 1999). Los Beraeidos por ejemplo, son poco abundantes y frecuentes, poseen una puntuación de 10 en el IBMWP y su presencia se limita a ríos pequeños y asociados a los musgos (o a las gravas $\mathrm{y}$ arenas). Por lo tanto, debido a que el número de taxones aumenta más allá de la fracción fija establecida sin variar el rango de calidad del IBMWP, se sugiere revisar el resto de la muestra un vez contados los 200 individuos en búsqueda de nuevos y raros taxones que podrían proporcionar información extra para estudios específicos en el caso de que fuera necesario.

Al comparar los dos protocolos de muestreo se observa como ambos no presentan diferencias en cuanto a número de familias, los índices IBMWP 
y IASPT, y por tanto, la selección de uno u otro aportarían un resultado similar, en condiciones de referencia. Sin embargo, en situaciones de referencia, la comunidad es mucho más diversa (Reynoldson et al., 1997) y el uso del Protocolo 1 significa que se pierde información ya que las especies raras o de pequeño tamaño no se detectan. Es por ello que, como los datos de las estaciones de referencia se usan para más análisis y no solamente para la calidad biológica (e.g. para establecer un método predictivo tipo RIVPACS en la segunda fase del proyecto), es necesario en estas estaciones el uso del Protocolo 2 mientras que para las localidades que no sean de referencia, el Protocolo 1 sería suficiente debido a la presencia de una comunidad sencilla, abundante y fácilmente reconocible, lo que ahorra mucho tiempo de separación y contaje (Countermanch, 1996). En ambos casos se muestrearan los hábitats reófilo y lenítico agrupados para obtener toda la comunidad presente.

El nivel taxonómico adecuado para la determinación de la calidad del agua ha sido ampliamente discutido y permite simplificar o no el PRECE, en función de los objetivos planteados (Resh \& Unzicker, 1975; Cranston, 1990; Marchant et al., 1995; Bowman \& Bailey, 1997). Mientras que utilizando niveles de identificación inferiores se gana en precisión (Furse et al., 1984; Resh et al., 1995), muchos son los estudios e índices que usan la familia como nivel taxonómico para determinar la calidad biológica (Armitage et al., 1987; AlbaTercedor \& Sánchez-Ortega, 1988; Corkum, 1989; Prat et al., 1999; Hewlett, 2000). Por otra parte, Stubauer \& Mogg (2000) apuntan que el uso de índices biológicos basados a nivel de familia puede suponer una importante pérdida de información sobre el efecto ambiental de la perturbación. A pesar de todo ello, varios estudios muestran que utilizando niveles taxonómicos superiores, los patrones de distribución que indican las comunidades son muy similares a los mostrados usando las especies (Furse et al., 1984; Ferrano \& Cole, 1992; Rutt et al., 1993; Marchant et al., 1995; Zamora-Muñoz \& Alba-Tercedor, 1996; Bowman \& Bailey, 1997; Nielsen et al., 1998). En este sentido, Bowman \& Bailey (1997) al comparar matri- ces de similaridad a nivel de género y familia, encuentran que en las estaciones perturbadas la correlación entre ambas es superior que en las de referencia. El índice IBMWP, utilizado en la metodología GUADALMED, está basado en la determinación de la calidad del agua a nivel de familia, y numerosos estudios en la Península inciden en su aplicabilidad y funcionalidad para detectar perturbaciones (Zamora-Muñoz et al., 1995; AlbaTercedor, 1996; Zamora-Muñoz \& Alba-Tercedor; 1996, García-Criado et al., 1999; Alba-Tercedor \& Pujante, 2000). No obstante, el uso del Protocolo 2 en la muestra de referencia permitirá, en el caso de que sea necesario, determinar las muestras a niveles taxonómicos inferiores, para con ello facilitar estudios sobre distribución espacial y temporal de especies a lo largo del Mediterráneo (Bonada, 2003) que podrán ser comparados con los que muestran las familias.

Los resultados obtenidos para el QBR muestran las diferencias que existen entre los puntos de muestreo cercanos, y ponen de relieve la dependencia de este índice de las condiciones locales, como ya se sabía a partir de su diseño (Munné et al., 1998). Debido a ello, y para que el índice se adecue mejor a las condiciones locales de cada cuenca de estudio, se modificará el índice original de las zonas más templadas (Munné et al. 1998) según la experiencia hallada en su aplicación en zonas más áridas (Suárez \& Vidal Abarca, 2000). Los resultados y aplicación en las cuencas muestreadas se exponen ampliamente en otra sección de este volumen. Otro hecho a destacar de la aplicación del QBR son las diferencias que existen a lo largo de un mismo punto de muestreo (diferencias que en nuestro caso se muestran con los distintos valores medios entre grupos). Ello tiene especial relevancia en la selección de condiciones de referencia donde, según la DMA, deberíamos buscar localidades con calidades biológicas del agua y de ribera muy buenas, lo que implicaría, en algunos casos, recorrer el río aguas arriba y abajo del punto de muestreo, para buscar un tramo donde el bosque de ribera esté mejor conservado sin que la calidad biológica del agua empeorase, y no limitar la determinación del QBR en el mismo punto de muestreo. 
A pesar de que Zamora-Muñoz et al., (1995) demostraron que los valores del IBMWP son independientes de la estacionalidad, hecho que sugeriría la simplificación de los muestreos anuales, en Furse et al. (1984) se muestra como los datos combinados de tres muestreos anuales (primavera, verano e invierno) categorizan mejor los ríos que uno solo. Así pues, para el proyecto GUADALMED se decidió hacer cuatro muestreos anuales (primavera, verano, otoño e invierno) para poder estudiar el efecto de los cambios temporales en los índices biológicos en los ríos mediterráneos muestreados, de una gran estacionalidad.

La intercalibración y selección de un protocolo de muestreo para evaluar el estado ecológico de un río, es un paso importante a tener en cuenta antes de empezar cualquier estudio a gran escala, ya que no todos los métodos son igualmente aplicables en cada zona y escala (Rosenberg \& Resh, 1993; Resh et al., 1995; Hill et al., 2000; Wrigth et al., 2000) y además, los objetivos bajo los cuales estos métodos fueron diseñados, pueden no ser los mismos (Barbour $e t$ al., 1999). El efecto del muestreador puede afectar enormemente la determinación del estado ecológico y por tanto a pesar de tratarse de una metodología sencilla, un mínimo aprendizaje sería necesario (Hannaford et al., 1997).

Con los resultados del ejercicio de intercalibración, el proyecto GUADALMED estableció el protocolo de muestreo siguiente, en que los puntos de referencia y no referencia se muestrearían de forma ligeramente diferente. Este protocolo fue el utilizado para todos los equipos del proyecto.

\section{PROTOCOLO GUADALMED}

(ver también Jáimez-Cuéllar, este volumen)

Protocolo 1. Puntos de NO referencia

- Seleccionar un tramo de estudio de $100 \mathrm{~m}$. Muestrear en todos los hábitats presentes con mangas de $250 \mu \mathrm{m}$

- Para los hábitats reófilos, se sitúa la manga a contracorriente y se remueve el sustrato inmediatamente aguas arriba con la ayuda de las botas, limpiando bien las piedras.

- Para los hábitats lénticos, se bate la vegetación lateral y se remueve el sustratos de gravas, arenas o sedimentos de las pozas.

- El contenido de las mangas se vierte en bateas de plástico blancas y se identifican a nivel de familia los organismos recolectados, indicando su rango de abundancia relativa: 1 (1-3 indv.), 2 (4-10 indv.), 3 (10-100 indv.) or 4 (>100 indv.).

- En caso de duda de algunos taxones se recogen 1-2 individuos que se conservan en viales con alcohol al $70 \%$ para su posterior identificación en el laboratorio.

- El muestreo se repite hasta que no se detecten nuevos taxones en sucesivas unidades de muestreo.

- Los índices biológicos IBMWP y IASPT se obtienen utilizando los taxones observados en el campo para todos los hábitats conjuntamente y los identificados en el laboratorio.

Protocolo 1. Puntos de referencia

- Seleccionar un tramo de estudio de $100 \mathrm{~m}$. Muestrear en todos los hábitats presentes con mangas de $250 \mathrm{~mm}$.

- Para los hábitats reófilos, se sitúa la manga a contracorriente y se remueve el sustrato inmediatamente aguas arriba con la ayuda de las botas, limpiando bien las piedras.

- Para los hábitats lénticos, se bate la vegetación lateral y se remueve el sustratos de gravas, arenas o sedimentos de las pozas.

- El contenido de las mangas se vierte en bateas de plástico blancas, se anotan los taxones recolectados. La muestra se vierte en botes de plástico, previamente etiquetados, y se conserva con alcohol al $70 \%$ o formol al $4 \%$.

- El muestreo se repite hasta que no se detecten nuevos taxones en sucesivas unidades de muestreo.

- En el laboratorio se separan e identifican 200 individuos al azar bajo la lupa binocular. Se revisa el resto de la muestra y se anotan los nuevos taxones no encontrados en los 200 individuos. Se asigna un rango de abundancia a cada uno de los taxones hallados en toda la muestra: 1 (1-3 indv.), 2 (4-10 indv.), 3 (10-100 indv.) or 4 (>100 indv.).

- Los índices biológicos IBMWP y IASPT se obtienen utilizando los taxones identificados en el laboratorio más aquellos observados en el campo pero no recolectados.

\section{Para todos los puntos}

- Muestrear 4 veces por año: primavera, verano, verano, otoño e invierno.

- Medir la temperatura, $\mathrm{pH}$, conductividad, oxígeno y caudal con los aparatos de campo.

- Tomar una muestra de agua para analizar en el laboratorio los parámetros químicos generales (a los que se refiere la DMA).

- Aplicar el índice QBR y el IHF.

- Identificar y cuantificar con un rango de abundancias relativas las macroalgas y macrófitos.

Figura 8. Pasos a seguir para el uso del Protocolo GUADALMED para la evaluación del estado ecológico de los ríos mediterráneos Guidelines for the use of the GUADALMED Protocol for the assessment of the ecological status of mediterranean rivers. 


\section{AGRADECIMIENTOS}

Este trabajo se ha realizado mediante la financiación de los proyectos HID98-0323-C05 y REN2001-3438-C07 del Ministerio de Ciencia y Tecnología y PLP/10/FS/97 de la Fundación Séneca de la CARM. Nuestro especial agradecimiento al Area de Coordinación y Aplicaciones Tecnológicas de la D.G.O.H. del Ministerio de Medio Ambiente, al Àrea de Medi Ambient de la Diputació de Barcelona, a la Delegación de Granada de la Consejería de Medio Ambiente de la Junta de Andalucía y a la Agencia Catalana del Aigua por su apoyo.

\section{BIBLIOGRAFÍA}

ABOAL, M. 1988. Aportación al conocimiento de las algas epicontinentales del S.E. de España. VII. Clorofíceas (Chlorophyceae Wille in Warming 1884). Conservatoire et Jardin Botaniques de Genève, 43:521-548.

ABOAL, M. 1989. Epilithic algal communities from River Segura Basin, Southeastern Spain. Arch. Hydrobiol., 116:113-124.

ALBA-TERCEDOR, J. \& A. SÁNCHEZ-ORTEGA. 1988. Un método rápido y simple para evaluar la calidad biológica de las aguas corrientes basado en el de Hellawell (1978). Limnetica, 4: 51-56.

ALBA-TERCEDOR, J. 1996. Macroinvertebrados acuáticos y calidad de las aguas de los ríos. $I V$ Simposio del Agua en Andalucía (SIAGA), Almería, España: 203-213.

ALBA-TERCEDOR, J. \& A. PUJANTE. 2000. Running-water biomonitoring in Spain. Opportunities for a predictive approach. In: Assessing the Biological Quality of Freshwater: RIVPACS and similar techniques. J.F. Wright, D.W. Sutcliffe \& M. Furse (eds.): 207-216. Freshwater Biological Association.

ARMITAGE, P. D., R. J. GUNN, M. T. FURSE, J. F. WRIGTH \& D. MOSS. 1987. The use of prediction to assess macroinvertebrate response to river regulation. Hydrobiologia, 144: 25-32.

BARBOUR, M. T. \& J. GERRITSEN. 1996. Subsampling of benthic samples: a defense of the fixed-count method. J. N. Am. Benthol. Soc., 15: 386-391.
BARBOUR, M. T., J. GERRITSEN, B. D. SNYDER \& J. B. STRIBLING. 1999. Rapid Bioassessment Protocols for Use in Streams and Wadeable Rivers: Periphyton, Benthic Macroinvertebrates and Fish, $2^{\text {ond }}$ ed. EPA 841-B-99-002. US EPA, Office of Water, Washington D.C., USA.

BLOCH, H. 1999. European water policy facing the new millennium: The EU Water Framework Directive. Assessing the Ecological Integrity of Running Waters, Vienna, Austria: 9-11.

BONADA, N. 2003. Ecology of the macroinvertebrate communities in mediterranean rivers at different scales and organization levels. Tesis Doctoral, Universidad de Barcelona. $355 \mathrm{pp}$

BOWMAN, M. F. \& R. C. BAILEY. 1997. Does taxonomic resolution affect the multivariate description of the structure of freshwater benthic macroinvertebrate communities? Can. J. Fish. Aquat. Sci., 54: 1802-1807.

CAO, Y. \& D. D. WILLIAMS. 1999. Rare species are important in bioassessment (Reply to the comment by Marchant). Limnol. Oceanogr., 44: 1840-1841.

CAO, Y., D. P. LARSEN \& R. ST. J. THORNE. 2001. Rare species in multivariate analysis for bioassessment: some considerations. J. N. Am. Benthol. Soc., 20: 144-153.

CARTER, J. L. \& S. V. FEND. 2001. Inter.-annual changes in the benthic community structure of riffles and pools in reaches of contrasting gradient. Hydrobiologia, 459: 187-200.

CARTER, J. L. \& V. H. RESH. 2001. After site selection and before data analysis: sampling, sorting, and laboratory procedures used in stream benthic macroinvertebrate monitoring programs by USA state agencies. J. N. Am. Benthol. Soc., 20: 658682.

CHESSMAN, B. C. \& D. P. ROBINSON. 1987. Some effects of the 1982-83 drougth on water quality and macroinvertebrate fauna in the lower La Trobe River, Victoria. Aust. J. Mar. Freshwat. Res., 38: 289-299.

CHOVAREC, A., P. JÄGER, M. JUNGWIRTH, V. KOLLER-KREIMEL, O. MOOG, S. MUHAR \& ST. SCHMUTZ. 2000. The Austrian way of assessing the ecological integrity of running waters: a contribution to the EU Water Framework Directive. Hydrobiologia, 422/423: 445-452.

CHUTTER, F. M. 1998. Research on the Rapid Biological Assessment of Water Quality Impacts in Streams and Rivers. Water Research Commision. WRC Report No. 422/1/98. 
CORKUM, L. D. 1989. Patterns of benthic invertebrate assemblages in rivers of northwestern North America. Freshwat. Biol., 21: 191-205.

COUNTERMANCH, D. L. 1996. Commentary on the subsampling procedures used for rapid bioassessments. J. N. Am. Benthol. Soc., 15: 381-385.

CRANSTON, P. S. 1990. Biomonitoring and invertebrate taxonomy. Environmental Monitoring and Assessment, 14: 265-273.

CUFFNEY, T. F., M. E. GURTZ \& M. R. MEADOR. 1993. Methods for collecting benthic invertebrate samples as part of the National Water-Quality Assessment Program, $1^{\text {st }}$ Edition. US Geological Survey, Raleigh, North Carolina, Open-File Report 93-406.

DAVIES, P. E. 1994. National River Processes and Management Program Monitoring River Health Initiative. River Bioassessment Manual Version 1.0. Department of the Environment, Sport and Territories, Canberra.

D.O.C.E. 2000. Directiva 2000/60/CE del Parlamento Europeo y del Consejo de 23 de octubre de 2000 por la que se establece un marco comunitario de actuación en el ámbito de la politica de aguas. D.O.C.E. L 327 de 22.12.00. 69 pp.

ELLIOT, J. M. \& P. A. TULLET. 1978. A bibliography of samplers for benthic invertebrates. Occ. Publ. Freshwat. Biol. Ass., 4: 1-61.

ELLIOT, J. M. \& P. A. TULLETT. 1983. A supplement to a bibliography of samplers for benthic invertebrates. Occ. Publ. Freshwat. Biol. Ass., 20: $1-27$.

FERRANO, S. P. \& F. A. COLE. 1992. Taxonomic level sufficient for assessing a moderate impact on macrobenthic communities in Puget Sound, Washington, USA. Can. J. Fish. Aquat. Sci., 49: 1184-1188.

FURSE, M. T., D. MOSS, J. F. WRIGTH \& P. D. ARMITAGE. 1984. The influence of seasonal and taxonomic factors on the ordination and classification of running-water sites in Great Britain and on the prediction of their macro-invertebrate communities. Freshwat. Biol., 14: 257-280.

GARCÍA CRIADO, F., A. TOMÉ, F. J. VEGA \& C. ANTOLÍN. 1999. Performance of some diversity and biotic indices in rivers affected by coal mining in northwestern Spain. Hydrobiologia, 394: 209-217.

GAUCH, H. G. 1982. Multivariate Analysis in Community Ecology. Cambridge: Cambridge University Press.
HANNAFORD, M. J., M. T. BARBOUR \& V. H. RESH. 1997. Training reduces observer variability in visual-base assessments of stream habitat. J. N. Am. Benthol. Soc., 16: 853-860.

HARPER, D. M., J. L. KEMP, B. VOGEL' \& M. D. NEWSON. 2000. Towards the assessment of "ecological integrity" in running waters of the United Kingdom. Hydrobiologia, 422/423: 133-142.

HEWLETT, R. 2000. Implications of taxonomic resolution and sample habitat for stream classification at a broad geographic scale. J. N. Am. Benthol. Soc., 19: 352-361.

HILL, M. O., D. B. ROY, J. O. MOUNTFORD \& R. G. H. BUNCE. 2000. Extending Ellenberg's indicator values to a new area: an algorithmic approach. J. Appl. Eco., 37: 3-15.

HULBERT, S. H. 1984. Pseudoreplication and the design of ecological field experiments. Ecological Monographs, 54: 187-211.

KARR, J. R. 1981. Assessment of biotic integrity using fish communities. Fisheries, 6: 21-27.

KARR, J. R. 1996. Ecological integrity and ecological health are not the same. In: Engineering Within Ecological Constraints. P.C. Schulze, (ed.): 97109. Washington, DC, National Academy Press.

KARR, J. R. \& E. W. CHU. 2000. Introduction: Sustaining living rivers. Hydrobiologia, 422/423: 1-14.

KERANS, B. L., J. R. KARR \& S. A. AHLSTEDT. 1992. Aquatic invertebrate assemblages: spatial and temporal differences among sampling protocols. J. N. Am. Benthol. Soc., 11: 377-390.

MARCHANT, R. 1990. Robustness of classification and ordenation techniques applied to macroinvertebrate communities from the La Trobe River, Victoria. Aust. J. Mar. Freshwat. Res., 41: 493-504.

MARCHANT, R., L. A. BARMUTA \& B. C. CHESSMAN. 1995. Preliminary study of the ordination of macroinvertebrate communities from running waters in Victoria, Australia. Aust. J. Mar. Freshwat. Res., 45: 945-962.

MARCHANT, R. 1999. How important are rare species in aquatic community ecology and bioassessment? A comment on the conclusions of Cao et al. Limnol. Oceanogr., 44: 1840-1841.

MCALEECE, N., P. J. D. LAMBSHEAD, G. L. J. PATERSON \& J. D. GAGE. 1997. Biodiversity Professional. London: The Natural History Museum \& The Scottish Association for Marine Science. 
CORKUM, L. D. 1989. Patterns of benthic invertebrate assemblages in rivers of northwestern North America. Freshwat. Biol., 21: 191-205.

COUNTERMANCH, D. L. 1996. Commentary on the subsampling procedures used for rapid bioassessments. J. N. Am. Benthol. Soc., 15: 381-385.

CRANSTON, P. S. 1990. Biomonitoring and invertebrate taxonomy. Environmental Monitoring and Assessment, 14: 265-273.

CUFFNEY, T. F., M. E. GURTZ \& M. R. MEADOR. 1993. Methods for collecting benthic invertebrate samples as part of the National Water-Quality Assessment Program, $1^{\text {st }}$ Edition. US Geological Survey, Raleigh, North Carolina, Open-File Report 93-406.

DAVIES, P. E. 1994. National River Processes and Management Program Monitoring River Health Initiative. River Bioassessment Manual Version 1.0. Department of the Environment, Sport and Territories, Canberra.

D.O.C.E. 2000. Directiva 2000/60/CE del Parlamento Europeo y del Consejo de 23 de octubre de 2000 por la que se establece un marco comunitario de actuación en el ámbito de la politica de aguas. D.O.C.E. L 327 de 22.12.00. 69 pp.

ELLIOT, J. M. \& P. A. TULLET. 1978. A bibliography of samplers for benthic invertebrates. Occ. Publ. Freshwat. Biol. Ass., 4: 1-61.

ELLIOT, J. M. \& P. A. TULLETT. 1983. A supplement to a bibliography of samplers for benthic invertebrates. Occ. Publ. Freshwat. Biol. Ass., 20: $1-27$.

FERRANO, S. P. \& F. A. COLE. 1992. Taxonomic level sufficient for assessing a moderate impact on macrobenthic communities in Puget Sound, Washington, USA. Can. J. Fish. Aquat. Sci., 49: 1184-1188.

FURSE, M. T., D. MOSS, J. F. WRIGTH \& P. D. ARMITAGE. 1984. The influence of seasonal and taxonomic factors on the ordination and classification of running-water sites in Great Britain and on the prediction of their macro-invertebrate communities. Freshwat. Biol., 14: 257-280.

GARCÍA CRIADO, F., A. TOMÉ, F. J. VEGA \& C. ANTOLÍN. 1999. Performance of some diversity and biotic indices in rivers affected by coal mining in northwestern Spain. Hydrobiologia, 394: 209-217.

GAUCH, H. G. 1982. Multivariate Analysis in Community Ecology. Cambridge: Cambridge University Press.
HANNAFORD, M. J., M. T. BARBOUR \& V. H. RESH. 1997. Training reduces observer variability in visual-base assessments of stream habitat. J. N. Am. Benthol. Soc., 16: 853-860.

HARPER, D. M., J. L. KEMP, B. VOGEL' \& M. D. NEWSON. 2000. Towards the assessment of "ecological integrity" in running waters of the United Kingdom. Hydrobiologia, 422/423: 133-142.

HEWLETT, R. 2000. Implications of taxonomic resolution and sample habitat for stream classification at a broad geographic scale. J. N. Am. Benthol. Soc., 19: 352-361.

HILL, M. O., D. B. ROY, J. O. MOUNTFORD \& R. G. H. BUNCE. 2000. Extending Ellenberg's indicator values to a new area: an algorithmic approach. J. Appl. Eco., 37: 3-15.

HULBERT, S. H. 1984. Pseudoreplication and the design of ecological field experiments. Ecological Monographs, 54: 187-211.

KARR, J. R. 1981. Assessment of biotic integrity using fish communities. Fisheries, 6: 21-27.

KARR, J. R. 1996. Ecological integrity and ecological health are not the same. In: Engineering Within Ecological Constraints. P.C. Schulze, (ed.): 97109. Washington, DC, National Academy Press.

KARR, J. R. \& E. W. CHU. 2000. Introduction: Sustaining living rivers. Hydrobiologia, 422/423: 1-14.

KERANS, B. L., J. R. KARR \& S. A. AHLSTEDT. 1992. Aquatic invertebrate assemblages: spatial and temporal differences among sampling protocols. J. N. Am. Benthol. Soc., 11: 377-390.

MARCHANT, R. 1990. Robustness of classification and ordenation techniques applied to macroinvertebrate communities from the La Trobe River, Victoria. Aust. J. Mar. Freshwat. Res., 41: 493-504.

MARCHANT, R., L. A. BARMUTA \& B. C. CHESSMAN. 1995. Preliminary study of the ordination of macroinvertebrate communities from running waters in Victoria, Australia. Aust. J. Mar. Freshwat. Res., 45: 945-962.

MARCHANT, R. 1999. How important are rare species in aquatic community ecology and bioassessment? A comment on the conclusions of Cao et al. Limnol. Oceanogr., 44: 1840-1841.

MCALEECE, N., P. J. D. LAMBSHEAD, G. L. J. PATERSON \& J. D. GAGE. 1997. Biodiversity Professional. London: The Natural History Museum \& The Scottish Association for Marine Science. 
MELLADO, A., M. L. SUÁREZ, J. L. MORENO \& M. R. VIDAL-ABARCA. 2002. Aquatic macroinvertebrate biodiversity in the Segura River basin (S.E. Spain). Ver. Internat. Verein. Limnol., 28: $1-6$

METZELING, L. \& J. MILLER. 2001. Evaluation of the sample size used for the rapid bioassessment of rivers using macroinvertebrates. Hydrobiologia, 444: 159-170.

MUNNÉ, A., C. SOLÀ \& N. PRAT. 1998. QBR: Un índice rápido para la evaluación de la calidad de los ecosistemas de ribera. Tecnología del Agua, 175: 20-37.

MUNNÉ, A., N. PRAT, C. SOLÀ, N. BONADA \& M. Rieradevall. 2003. A simple field method for assessing the ecological quality of riparian habitat in rivers and streams. QBR index. Aquatic Conserv.: Mar. Freshw. Ecosyst., 13: 147-164.

NIELSEN, D. L., R. J. SHIEL \& F. J. SMITH. 1998. Ecology versus taxonomy: is there a middle ground? Hydrobiologia, 387/388: 451-457.

NORRIS, R.H. 1995. Biological monitoring: the dilemma of data analysis. J. N. Am. Benthol. Soc., 14: 440-450.

PARDO, I., M. ÁLVAREZ, J. CASAS, J. L. MORENO, S. VIVAS, N. BONADA, J. ALBA-TERCEDOR, P. JÁIMEZ-CUÉLLAR, G. MOYÀ, N. PRAT, S. ROBLES, M. L. SUÁREZ, M. TORO \& M. R. VIDAL-ABARCA. 2004. El hábitat de los ríos mediterráneos. Diseño de un índice de diversidad de hàbitat. Limnetica, 21: 115-132

PLAFKIN, J. L., M. T. BARBOUR, K. D. PORTER, S. K. GROSS \& R. M. HUGHES. 1989. Rapid Bioassessment protocols for use in streams and rivers: benthic macroinvertebrates and fish. EPA/444/4-89-001. United Estates Environmental Protection Agency, Washington D. C., USA.

PRAT, N. 1993. El futuro de los ríos españoles: secos o contaminados. Quercus, 84: 22-24

PRAT, N., M. RIERADEVALL, A. MUNNÉ, C. SOLÀ \& G. CHACÓN. 1997. La qualitat ecológica del Llobregat, el Besòs i el Foix. Informe 1996. Barcelona: Diputació de Barcelona. Àrea de Medi Ambient (Estudis de la Qualitat Ecológica dels Rius, 2).

PRAT, N., M. RIERADEVALL, A. MUNNÉ, C. SOLÀ \& N. BONADA. 1999. La qualitat ecológica del Llobregat, el Besòs i el Foix. Informe 1997. Barcelona: Diputació de Barcelona. Àrea de Medi Ambient (Estudis de la Qualitat Ecológica dels Rius, 6).
RESH, V. H. \& J. D. UNZICKER. 1975. Water quality monitoring and aquatic organisms: the importance of species identification. J. Water Pollut. Control Fed., 47: 9-19.

RESH, V. H. \& J. K. JACKSON. 1993. Rapid assessment approaches to biomonitoring using benthic macroinvertebrates. In: Freshwater Biomonitoring and Benthic Macroinvertebrates. D.M. Rosenberg \& V.H. Resh (eds.): 195-233. Chapman and Hall, New York, USA.

RESH, V. H., R. H. NORRIS \& M. T. BARBOUR. 1995. Design and implementation of rapid assessment approaches for water resource monitoring using benthic macroinvertebrates. Aust. J. Ecol., 20: $108-121$.

REYNOLDSON, T. B., R. H. NORRIS, V. H. RESH, K. E. DAY \& D. M. ROSENBERG. 1997. The reference condition: a comparision of multimetric and multivariate approaches to assess water-quality impairment using benthic macroinvertebrates. J. N. Am. Benthol. Soc., 16: 833-852.

RICO, E., A. RALLO, M. A. SEVILLANO \& M. L. ARRETXE. 1992. Comparision of several biological indices based on macroinvertebrate benthic community for assessment of running water quality. Annls. Limnol., 28: 147-156.

RIERADEVALL, M., N. BONADA \& N. PRAT. 1999. Community structure and water quality in the mediterranean streams of a natural park (St. Llorenç del Munt, NE Spain). Limnetica, 17: 4556.

ROSENBERG, D. M. 1978. Practical sampling of freshwater macrozoobenthos: a bibliography of useful texts, reviews, and recent papers. Can. Fish. Mar. Serv. Tech. Rep., 790: 1-15.

ROSENBERG, D. M. \& V. H. RESH. 1993. Freshwater Biomonitoring and Benthic Macroinvertebrates. New York: Chapman \& Hall.

RUTT, G. P., T. D. PICKERING \& N. R. REYNOLDS. 1993. The impact of livestock farming on Welsh streams: The development and testing of a rapid biological method for use in the assessment and control of organic pollution from farms. Envir. Pollut., 81: 217-228.

SMITH, M. J., W. R. KAY, D. H. D. EDWARD, P. J. PAPAS, K. ST. J. RICHARDSON, J. C. SIMPSON, A. M. PINDER, D. J. CALE, P. H. J. HORWITZP, J. A. DAVIS, F. H. YUNG, R. H. NORRIS $\&$ S. A. HALSE. 1999. AusRivAS: using macroinvertebrates to assess ecological condition of rivers in Western Australia. Freswat. Biol., 41: 269-282. 
STATSOFT, INC. 1999. STATISTICA for Windows (Computer Program Manual). Tulsa: OK.

STOREY, A. W., D. H. D. EDWARD \& P. GAZEY. 1991. Surber and kick sampling: a comparision for the assessment of macroinvertebrate community streams of SW Australia. Hydrobiologia, 211: 111-121.

STUBAUER, I. \& O. MOOG. 2000. Taxonomic sufficiency versus need of information - comments based on the Austrian experiences in biological water quality monitoring. Verh. Int. Verein. Limnol., 27: 2562-2566.

SUÁREZ, M. L. \& M. R. VIDAL-ABARCA. 2000. Aplicación del índice de calidad del bosque de ribera, QBR (Munné et al., 1998) a los cauces fluviales de la cuenca del río Segura. Tecnología del Agua, 201: 33-45.

SUÁREZ, M. L., M. R. VIDAL-ABARCA, M. M. SÁNCHEZ-MONTOYA, J. ALBA-TERCEDOR, M. ÁlVAREZ, J. AVILÉS, N. BONADA, J. CASAS, P. JÁIMEZ-CUÉLLAR, A. MUNNÉ, I. PARDO, N. PRAT, M. RIERADEVALL, M. J. SALINAS, M. TORO \& S. VIVAS. 2002. Las riberas de los ríos mediterráneos y su calidad: El uso del índice QBR. Limnetica, 21: 135-148

TILLER, D. \& L. H. METZELING. 1998. Rapid Bioassessment of Victorian Streams. Victoria: EPA publication 604, Environment Protection Authory.

TORO, M., S. ROBLES, J. AVILÉS, C. NUÑO, S. VIVAS, N. BONADA, N. PRAT, J. ALBATERCEDOR, J. CASAS, C. GUERRERO, P. JÁIMEZ-CUÉLLAR, J. L. MORENO, G. MOYÁ, G. RAMON, M. L. SUÁREZ, M. R. VIDALABARCA, M. ÁlVAREZ \& I. PARDO. 2002. Calidad de las aguas de los ríos mediterráneos del proyecto GUADALMED. Limnetica, 21: 63-75
VVAA. 1988. Estudio e interrelaciones de los niveles de calidad biológica y físico-química de las aguas de la Cuenca del Segura. Origenes de la contaminación y bases para su planificación. Proyecto LUCDEME. Convenio ICONAUniversidad de Murcia.

VIDAL-ABARCA, M.R. 1985. Las aguas continentales de la Cuenca del Río Segura (SE. de España). Caracterización físico-química en relación al medio físico y humano. Tesis Doctoral, Universidad de Murcia.

VINSON, M.R. \& C.P. HAWKINS. 1996. Effects of sampling area and subsampling procedure on comparisons of taxa richness among streams. $J$. N. Am. Benthol. Soc., 15: 392-399.

WRIGTH, J.F., D. MOSS, P.D. ARMITAGE. \& M.T. FURSE. 1984. A preliminary classification of running water sites in Great Britain based on macroinvertebrate species and the prediction of community type using environmental data. Freshwat. Biol., 14:221-256.

WRIGTH, J.F., D.W. SUTCLIFFE \& M.T. FURSE. 2000. Assessing the biological quality of fresh waters: RIVPACS and other techniques. Freshwater Biological Association. Ambleside, UK

ZAMORA-MUÑOZ, C., C.E. SAINZ-CANTERO, A. SÁNCHEZ-ORTEGA \& J. ALBA-TERCEDOR. 1995. Are biological indices IBMWP and IASPT and their significance regarding water quality seasonality dependent? Factors explaining their variations. Wat. Res., 29: 285-290.

ZAMORA-MUÑOZ, C. \& J. ALBA-TERCEDOR. 1996. Bioassessment of organically polluted Spanish rivers, using a biotic index and multivariate methods. J. N. Am. Benthol. Soc., 15: 332-352. 
Anexo 1. Listado de taxones hallados en el tramo de río muestreado. List of taxa found in the sampling reach of the studied river.

\section{OLIGOCHAETA}

\begin{tabular}{|c|c|}
\hline ACHAETA & $\begin{array}{l}\text { Erpobdellidae } \\
\text { Glossiphoniidae }\end{array}$ \\
\hline TURBELLARIA & Planariidae \\
\hline CRUSTACEA & Echinogammarus sp. \\
\hline MOLLUSCA & $\begin{array}{l}\text { Lymnaea truncatula } \\
\text { Lymnaea peregra } \\
\text { Physella acuta } \\
\text { Hydrobiidae } \\
\text { Potamopyrgus jenkinsi }\end{array}$ \\
\hline EPHEMEROPTERA & $\begin{array}{l}\text { Alainites muticus } \\
\text { Baetis pavidus } \\
\text { Baetis rhodani } \\
\text { Cloeon dipterum } \\
\text { Procloeon bifidum } \\
\text { Caenis luctuosa } \\
\text { Ecdyonurus gr. ruffi-wautieri }\end{array}$ \\
\hline PLECOPTERA & $\begin{array}{l}\text { Nemouridae } \\
\text { Perlidae }\end{array}$ \\
\hline COLEOPTERA & $\begin{array}{l}\text { Nebrioporus clarki } \\
\text { Deronectes hispanicus } \\
\text { Bidessus minutissimus } \\
\text { Graptodytes fractus } \\
\text { Agabus gr. brunneus } \\
\text { Agabus ddymus } \\
\text { Agabus biguttatus } \\
\text { Hidroporus discretus } \\
\text { Lacophilus hyalinus } \\
\text { Lacophilus minutus } \\
\text { Haliplus lineatocollis } \\
\text { Haliplus mucronatus } \\
\text { Anacaena limbata } \\
\text { Anacaena globulus } \\
\text { Helochares lividus } \\
\text { Laccobius gracilis } \\
\text { Laccobius hispanicus } \\
\text { Ochthebius quadrifoveolatus } \\
\text { Ochthebius marinus } \\
\text { Limnebius maurus } \\
\text { Limnius volkmari } \\
\text { Dryops gracilis } \\
\text { Pomatinus substriatus } \\
\text { Helophorus flavipes } \\
\text { Elmis mauguetti } \\
\text { Hydrocyphon sp. } \\
\text { Elodes sp. }\end{array}$ \\
\hline
\end{tabular}

\begin{tabular}{|c|c|}
\hline ODONATA & $\begin{array}{l}\text { Pyrrhosoma nymphula } \\
\text { Orthetrum coerulescens } \\
\text { Orthetrum cf. cancellatum } \\
\text { Cordulegaster annulatus } \\
\text { Onychogomphusforcipatus } \\
\text { Onychogomphus uncatus } \\
\text { Anax imperator } \\
\text { Boyeria irene }\end{array}$ \\
\hline HETEROPTERA & $\begin{array}{l}\text { Naucoris maculatus } \\
\text { Notonecta maculata } \\
\text { Sigara nigronileata } \\
\text { Nepa cinerea } \\
\text { Microvelia pygmaea } \\
\text { Hydrometra stagnorum }\end{array}$ \\
\hline TRICHOPTERA & $\begin{array}{l}\text { Rhyacophila gr. munda } \\
\text { Agapetus sp. } \\
\text { Hydropsyche gr. pellucidula } \\
\text { Stenophylax sp. } \\
\text { Plectrocnemia } \text { sp. } \\
\text { Tinodes waeneri } \\
\text { Sericostomatidae } \\
\text { Mesophylax aspersus } \\
\text { Hydroptila vectis }\end{array}$ \\
\hline DIPTERA & $\begin{array}{l}\text { Tanypodinae } \\
\text { Tanytarsini } \\
\text { Orthocladiinae } \\
\text { Corynoneurinae } \\
\text { Quironomini } \\
\text { Athericidae } \\
\text { Limoniidae } \\
\text { Dixidae } \\
\text { Psychodidae } \\
\text { Tipulidae } \\
\text { Ceratopogonidae } \\
\text { Tabanidae } \\
\text { Simuliidae } \\
\text { Stratiomyidae } \\
\text { Ephydridae }\end{array}$ \\
\hline
\end{tabular}


Anexo 2. Versión original del índice de hábitat. Original version of the Habitat Index.

Calidad del hábitat fluvial

\begin{tabular}{|c|c|c|c|c|c|c|}
\hline Tipo delecho & $\%$ & $\mathbf{P}$ & \multirow[b]{2}{*}{ Elementos de heterogeneidad } & \multicolumn{3}{|c|}{ Puntuación } \\
\hline \multirow{4}{*}{$\begin{array}{l}\text { Consolidado } \\
\text { No consolidado } \\
\text { Artificial }\end{array}$} & $>50$ & 1 & & Aislado & Manchas & Dominante \\
\hline & $>50$ & 0 & & & & \\
\hline & $>50$ & -1 & Raíces expuestas & 0 & 1 & 0 \\
\hline & & & Troncos, ramas caídas & 0 & 1 & 0 \\
\hline Estructura & $\%$ & $\mathbf{P}$ & Hojarasca acumulada & 0 & 2 & 0 \\
\hline Pozas & $>60$ & 0 & Diques naturales & 0 & 1 & 0 \\
\hline \multirow{3}{*}{$\begin{array}{l}\text { Rápidos } \\
\text { Pozas y rápidos }\end{array}$} & $>60$ & 1 & Vegetación acuática & Aislado & Manchas & Dominante \\
\hline & $40 / 60$ & 2 & & & & \\
\hline & & & Macrófitas & & & \\
\hline \multirow[t]{2}{*}{ Cobertura de sombra } & $\%$ & $\mathbf{P}$ & Caráceas & 0 & 2 & 1 \\
\hline & & & Potamogeton sp. & 0 & 1 & -1 \\
\hline Porcentaje entre & $40-60$ & 1 & Otros macrófitos sumergidos & 0 & 1 & 0 \\
\hline \multirow[t]{2}{*}{ Substrato } & $\%$ & $\mathbf{P}$ & Macroalgas & & & \\
\hline & & & Cladophora sp. /Zignematales & 0 & 1 & -1 \\
\hline Lasca & $>10$ & 1 & Enteromorpha sp. & 0 & -1 & -2 \\
\hline Bloques & $>10$ & 1 & & & & \\
\hline Piedras & $>10$ & 1 & Biofilm & & & \\
\hline Cantos & $>10$ & 1 & Diatomeas & 0 & 2 & -1 \\
\hline Gravas & $>10$ & 1 & Cianofíceas & 0 & -1 & -2 \\
\hline Arenas & $>10$ & 1 & Musgos & 0 & 2 & 1 \\
\hline Limos & $>10$ & 1 & & & & \\
\hline
\end{tabular}

Puntuación final (de 0 a 25) (negativos $=0$ ) 